\title{
Role of Kinase Suppressor of Ras-1 in Neuronal Survival Signaling by Extracellular Signal-Regulated Kinase 1/2
}

\author{
Erzsebet Szatmari, ${ }^{1,2}$ Katarzyna B. Kalita, ${ }^{1,2}$ Giorgi Kharebava, ${ }^{1,2}$ and Michal Hetman ${ }^{1,2,3}$ \\ ${ }^{1}$ Kentucky Spinal Cord Injury Research Center and Departments of ${ }^{2}$ Neurological Surgery and ${ }^{3}$ Pharmacology and Toxicology, University of Louisville, \\ Louisville, Kentucky 40292
}

\begin{abstract}
Scaffolding proteins including kinase suppressor of Ras-1 (KSR1) determine specificity of signaling by extracellular signal-regulated kinase $1 / 2$ (ERK1/2), enabling it to couple diverse extracellular stimuli to various cellular responses. The scaffolding protein(s) that contributes to ERK1/2-mediated neuronal survival has not yet been identified. In cultured rat cortical neurons, BDNF activates ERK1/2 to enhance neuronal survival by suppressing DNA damage- or trophic deprivation-induced apoptosis. Here we report that in this system, BDNF increased KSR1 association with activated ERK1/2, whereas KSR1 knockdown with a short hairpin (sh) RNA reduced BDNFmediated activation of ERK1/2 and protection against a DNA-damaging drug, camptothecin (CPT). In contrast, BDNF suppression of trophic deprivation-induced apoptosis was unaffected by shKSR1 although blocked by shERK1/2. Also, overexpression of KSR1 enhanced BDNF protection against CPT. Therefore, KSR1 is specifically involved in antigenotoxic activation of ERK1/2 by BDNF. To test whether KSR1 contributes to ERK1/2 activation by other neuroprotective stimuli, we used a cAMP-elevating drug, forskolin. In cortical neurons, ERK1/2 activation by forskolin was protein kinase A (PKA) dependent but TrkB (receptor tyrosine kinase B) independent and was accompanied by the increased association between KSR1 and active ERK1/2. Forskolin suppressed CPT-induced apoptosis in a KSR1 and ERK1/2-dependent manner. Inhibition of PKA abolished forskolin protection, whereas selective PKA activation resulted in an ERK1/2- and KSR1-mediated decrease in apoptosis. Hence, KSR1 is critical for the antiapoptotic activation of ERK1/2 by BDNF or cAMP/PKA signaling. In addition, these novel data indicate that stimulation of cAMP signaling is a candidate neuroprotective strategy to intervene against neurotoxicity of DNA-damaging agents.
\end{abstract}

Key words: BDNF; cAMP; ERK1/2; KSR1; DNA damage; apoptosis

\section{Introduction}

Extracellular signal-regulated kinase 1 (ERK1) and ERK2 are protein kinases contributing to an evolutionary conserved signal transduction pathway that regulates cell proliferation, differentiation, survival, and nervous system plasticity (Pearson et al., 2001). The ERK1/2 pathway is activated by numerous extracellular signals and transduces them to diverse effector mechanisms. In neurons, the examples include ERK1/2-mediated couplings between neurotrophins and neurite outgrowth, neuronal activity patterns and synaptic plasticity/memory formation, and neurotrophins and suppression of DNA damage-induced apoptosis (for review, see Kaplan and Miller, 2000; Adams and Sweatt, 2002; Markus et al., 2002; Hetman and Gozdz, 2004). A major challenge to the field of neuronal signal transduction is identifi-

Received Feb. 12, 2007; accepted Aug. 16, 2007

This work was supported by National Institutes of Health Grant NS047341-01, The Commonwealth of Kentucky Challenge for Excellence, and Norton Healthcare. We thank Drs. Scott R. Whittemore and Theo Hagg for critical reading of this manuscript. Drs. Deborah K. Morrison, Moses V. Chao, Stanley McKnight, Theo Hagg, and Jacek Jaworski provided reagents used in this study.

Correspondence should be addressed to Michal Hetman, Kentucky Spinal Cord Injury Research Center, University of Louisville, 511 South Floyd Street, MDR616, Louisville, KY 40292. E-mail: michal.hetman@louisville.edu.

E. Szatmari's present address: Department of Neurobiology, Box 3209, Duke University Medical Center, Durham, NC 27710.

DOI:10.1523/JNEUROSCI.3473-07.2007

Copyright $\odot 2007$ Society for Neuroscience 0270-6474/07/2711389-12\$15.00/0 cation of the mechanism(s) that determines specificity in ERK1/2 signaling.

Scaffolding proteins provide one of the mechanisms contributing to specificity in kinase signaling cascades, including ERK1/2 (Morrison and Davis, 2003). These proteins ensure efficient and specific transduction of signals by physical binding and bringing together the upstream and downstream elements of signaling pathways. Kinase suppressor of Ras 1 (KSR1) is an ERK1/2 scaffolding protein, the splice variant (B-KSR1) of which is highly expressed in the brain, including the cerebral cortex (Muller et al., 2000; Giblett et al., 2002). In PC12 cells that were stimulated with NGF, an overexpressed KSR1 contributed to ERK1/2 activation and enhanced NGF effects on neurite outgrowth (Muller et al., 2000). Shalin et al. (2006) recently reported that some forms of hippocampal synaptic plasticity and hippocampus-dependent memory were impaired in the absence of KSR1. Thus, KSR1 may contribute to ERK1/2-mediated neuronal differentiation and plasticity.

In addition to its involvement in neurite outgrowth or neuronal plasticity, ERK1/2 regulates neuronal survival. ERK1/2 activation by NGF or BDNF suppresses DNA damage-induced apoptosis in sympathetic or cortical neurons, respectively (Anderson and Tolkovsky, 1999; Hetman et al., 1999; Gozdz et al., 2003). Also, in cerebellar granule neurons, ERK1/2 activation by either BDNF or cAMP elevation reduces trophic deprivation (TD)- 
induced apoptosis (Villalba and Journot, 1997; Bonni et al., 1999). Finally, ERK1/2 is critical for neuroprotection by combined treatment with BDNF and cAMP-elevating agents in trophic-deprived retinal ganglion cells (Meyer-Franke et al., 1998) or by moderate stimulation of glutamate NMDA receptors (NMDARs) in cortical or cerebellar granule neurons challenged with hypoxic/excitotoxic insults (Gonzalez-Zulueta et al., 2000; Lee et al., 2005; Zhu et al., 2005). The scaffolding protein(s) that mediates the prosurvival activity of ERK $1 / 2$ in neurons has not yet been identified.

DNA damage occurs in numerous neurodegenerative conditions and contributes to neurotoxicity of anticancer therapies (Rolig and McKinnon, 2000; Geller et al., 2001; Kruman et al., 2002; Koh et al., 2005; McDonald et al., 2005; Wang et al., 2005). Identification of survival signaling pathways, the protective effects of which against DNA damage could be enhanced pharmacologically, may provide useful strategies to increase the therapeutic index of anticancer treatments. The signaling by cAMP offers numerous opportunities for pharmacological interventions with some of them showing neuroprotective effects in experimental models of ischemia or spinal cord injury (McCullough et al., 2004; Pearse et al., 2004). Studies evaluating a possibility that increased cAMP can protect against the neurotoxicity of DNA damage have not been reported before.

This study was designed to test whether in cultured rat cortical neurons (1) KSR1 may be involved in BDNF-mediated activation of ERK1/2, (2) KSR1 plays a role in ERK1/2-mediated neuroprotection against apoptosis, and (3) cAMP activates antiapoptotic signaling by KSR1-ERK1/2.

\section{Materials and Methods}

Materials. The following plasmids have been described previously: pON260 (Cherrington and Mocarski, 1989), wild-type MKP3 (Groom et al., 1996), wild-type protein kinase inhibitor $\alpha$ (PKI $\alpha$ ) (Wiley et al., 1999), wild-type KSR1 (WT-KSR1) (Cacace et al., 1999), pcDNA3-FlagBCL-2 (del Peso et al., 1997), and pSUPER vector (Brummelkamp et al., 2002). An shRNA construct based on the pSUPER shRNA expression vector and targeting green fluorescent protein (GFP) was donated by Dr. Jacek Jaworski (International Institute of Molecular and Cell Biology, Warsaw, Poland). The following antibodies and reagents were obtained from commercial sources: mouse anti-KSR1 antibody (C3H7; Upstate Biotechnology, Lake Placid, NY); rabbit anti-phospho-ERK1/2 antibody (pThr183/pTyr185, anti-ACTIVE MAPK; Promega, Madison, WI); mouse anti-ERK1/2 antibody; rabbit anti-phospho-MEK/MKK1/2 antibody (pSer217/221); rabbit anti-MEK/MKK1/2 antibody; rabbit antiphospho-protein kinase A (PKA) subtrate antibody (Cell Signaling Technology, Beverly, MA); rabbit anti- $\beta$-galactosidase (anti- $\beta$-gal; Invitrogen, Carlsbad, CA); mouse anti-flag antibody and mouse and rabbit anti- $\beta$-actin antibodies (Sigma, St. Louis, MO); rabbit anti-KSR1 and anti-receptor tyrosine kinase (Trk) C14 antibodies (Santa Cruz Biotechnology, Santa Cruz, CA); HRP-labeled anti-mouse or anti-rabbit IgG antibody for Western blot analysis; Texas Red-conjugated goat antirabbit IgG antibody for immunofluorescence (Calbiochem, La Jolla, CA); BDNF, forskolin, camptothecin (CPT), H89, myrPKI, dizocilpine maleate (MK-801), U0126, and Sp-cAMP (Sigma or Calbiochem). Rabbit anti-KSR1 antibody was donated by Dr. Deborah K. Morrison (NCIFrederick, Frederick, MD). Rabbit anti-phospho-TrkB antibody that was raised against a phospho-peptide corresponding to the phospholipase C- $\gamma$ binding site of rat TrkB (Y816) was donated by Dr. Moses V. Chao (Skirball Institute, New York, NY).

Generation of shRNA expression constructs. To generate KSR1 shRNA constructs, the rat KSR1 mRNA (XM_340852) sequence was analyzed using shRNA design software (http://sonnhammer.cgb.ki.se/). Three sequences corresponding to nucleotides 5072-5090, 5330-5348, and 5421-5439 were selected. Comparison of mouse and rat mRNA sequences of KSR1 demonstrated that the selected regions were completely conserved between these species, indicating feasibility of validation ex- periments with overexpressed mouse KSR1. Oligonucleotides (sequence 1, gatccccacctgaagctgttcaagaattcaagagattcttgaacagcttcaggttttttggaaa; sequence 2, gatcccccaagaatgtcttctatgattcaagagatcatagaagacattcttggtttttggaaa; sequence 3 , gatccccgaaccaactgaaactgtcattcaagagatgacagtttcagttggttctttttggaaa) were designed together with their complementary counterparts, annealed, and subcloned into PSUPER vector digested with BglII and HindIII (Brummelkamp et al., 2002). Except for the validation experiment that evaluated each individual siKSR1 construct (Fig. $2 B$ ), a mix of all three plasmids (1:1:1) was used in all other studies. ERK1/2 short hairpin (sh) RNAs were generated in a similar way to those against KSR1. We used the rat counterparts of mouse ERK1/2 sequences that were selected and validated by others (Yun et al., 2005; Vantaggiato et al., 2006). Oligonucleotide sequences for cloning shRNA constructs were as follows: shERK1-1, gatccccatgtcataggcatccgagattcaagagatctcggatgcctatgacattttttggaaa; shERK2-1, gatccccgaccggatgttaacctttattcaagagataaaggttaacatccggtctttttggaaa; shERK1-2, gatccccagttcgagttgctatcaagttcaagagacttgatagcaactcgaacttttttggaaa; shERK2-2, gatccccgtacagagctccagaaattttcaagagaaatttctggagctctgtactttttggaaa. All these shRNAs effectively and specifically inhibited expression of their respective targets (G. Kharebava, K. B. Kalita, and M. Hetman, unpublished observations). Data presented in Figure 3, $A$ and $B$, were obtained with 1:1 mix of shERK1-1 and shERK2-1.

Cell culture and transfections. Cortical neurons were prepared from newborn Sprague Dawley rats at postnatal day 0 as described previously (Habas et al., 2006). Briefly, culture medium was basal medium Eagle (BME) supplemented with 10\% heat-inactivated bovine calf serum (HyClone, Logan, UT), $35 \mathrm{~mm}$ glucose, $1 \mathrm{~mm}$ L-glutamine, $100 \mathrm{U} / \mathrm{ml}$ penicillin, and $0.1 \mathrm{mg} / \mathrm{ml}$ streptomycin. Cytosine arabinoside $(2.5 \mu \mathrm{M})$ was added to the cultures on the second day after seeding [ $2 \mathrm{~d}$ in vitro (DIV2)] to inhibit the proliferation of non-neuronal cells. The cells were used for experiments at DIV6-7, unless indicated otherwise. Transient transfections were performed on DIV3 or DIV4 using the Lipofectamine2000 (Invitrogen) as described previously (Hetman et al., 2002). Electroporation of freshly dissociated newborn rat cortical neurons was conducted using the rat neuron nucleofection kit (Amaxa, Köln, Germany).

Drug treatments. BDNF was diluted in PBS containing $0.1 \%$ bovine serum albumin before addition to the cells. U0126, forskolin, Sp-8pCPT-cAMPS (Sp), and CPT were dissolved in dimethyl sulfoxide (DMSO), whereas H89 and myrPKI were dissolved in water. The final concentration of DMSO in the medium was $0.2-0.4 \%$. All inhibitors were added $30 \mathrm{~min}$ before BDNF or cAMP signaling activators. The protective treatments with BDNF or forskolin or Sp were initiated $30 \mathrm{~min}$ before adding CPT. For Western blot analysis, stimulation with forskolin or $\mathrm{Sp}$ was performed in cultured medium with reduced serum content (1\%). For KSR1/phosphorylated, active ERK (pERK) immunofluorescence studies, neurons were placed in TD medium for $1 \mathrm{~h}$ before adding BDNF.

Immunohistochemistry. Naive adult female Sprague Dawley rats (2-3 months old; weight, 180-220 g; Harlan, Indianapolis, IN) were housed in groups under a $12 \mathrm{~h}$ light/dark cycle and with ad libitum access to water and food. All animals were treated in accordance with the guidelines of the National Institutes of Health Guide for the Care and Use of Laboratory Animals and the University of Louisville guidelines for the care and use of laboratory animals. Anesthetized animals were perfused transcardially, and free-floating $30-\mu \mathrm{m}$-thick coronal brain sections were prepared after postfixation. For staining, rabbit polyclonal anti-KSR1 antibody (1:100 in PBS/0.3\% Triton X-100; Santa Cruz Biotechnology) was applied overnight at $4^{\circ} \mathrm{C}$, followed by incubation with biotinylated anti-rabbit antibody (1:500) and streptavidine-Alexa 488 conjugate (1:100) (Invitrogen).

Immunocytochemistry. Transfected neurons were detected by immunostaining for $\beta$-gal using standard technique as described previously (Hetman et al., 1999). To visualize the nuclei of transfected cells, counterstaining with Hoechst 33258 was performed. For KSR1/pERK immunostaining, neurons rabbit anti-KSR1 (Santa Cruz Biotechnology) and mouse anti-phospho-Thr ${ }^{183} /$ Tyr $^{185}$ pERK1/2 (Cell Signaling Technology) were used. Cells were fixed with $4 \%$ paraformaldehyde and incubated in methanol for $10 \mathrm{~min}$ at $-20^{\circ} \mathrm{C}$ followed by standard blocking buffer (5\% goat serum in PBS/0.3\% Triton X-100; for $1 \mathrm{~h}$ at room temperature). Primary antibodies KSR1 (1:100) and pERK1/2 (1:200, diluted 
A
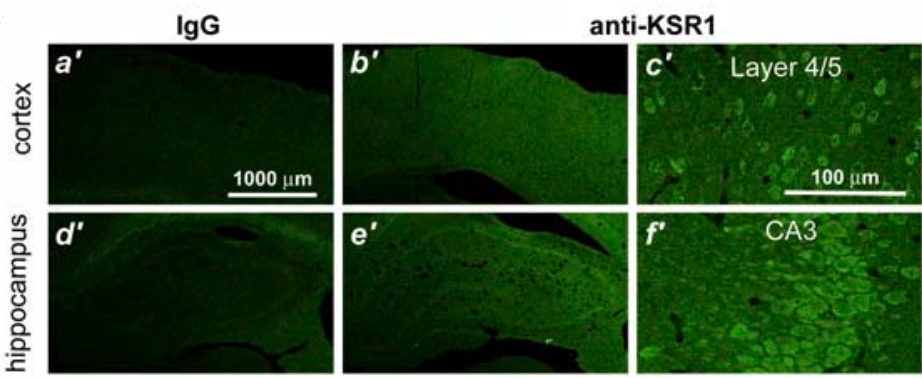

B
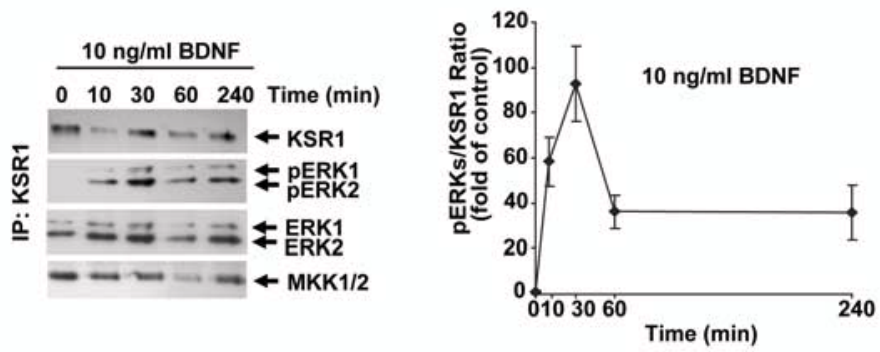

C
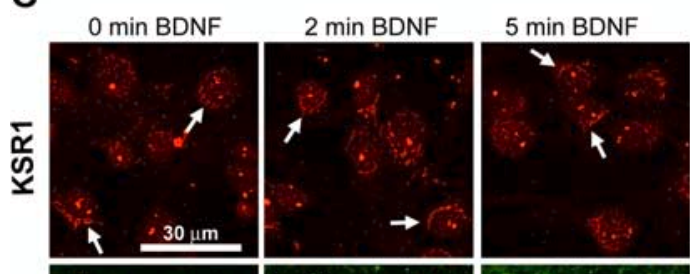

D
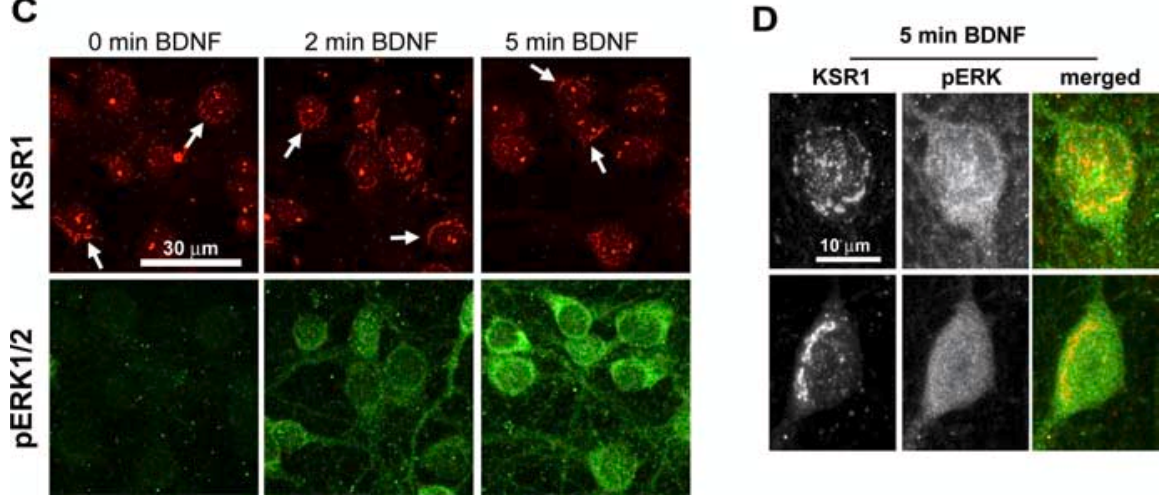

Figure 1. KSR1 associates with active ERK1/2 in BDNF-stimulated rat cortical neurons. $A, K S R 1$ expression in the adult rat brain. KSR1 immunoreactivity in coronal rat brain sections was visualized by immunofluorescence with a rabbit anti-KSR1 antibody ( $\boldsymbol{b}^{\prime}$, $\left.\boldsymbol{c}^{\prime}, \boldsymbol{e}^{\prime}, \boldsymbol{f}^{\prime}\right)$; IgG from nonimmunized rabbits was used to control the specificity of staining $\left(\boldsymbol{a}^{\prime}, \boldsymbol{d}^{\prime}\right)$. Low-magnification views of the neocortex $\left(\boldsymbol{a}^{\prime}, \boldsymbol{b}^{\prime}\right)$ and the hippocampus $\left(\boldsymbol{d}^{\prime}, \boldsymbol{e}^{\prime}\right)$ are shown. In $\boldsymbol{c}^{\prime}$ and $\boldsymbol{f}^{\prime}$, higher-magnification views of the neocortical layers $4 / 5$ and the $C A 3$ subfield of the hippocampus are presented as indicated. Single confocal sections are shown; a similar pattern of KSR1 staining was observed in sections from four different animals. B, Coimmunoprecipitation of KSR1 and active ERK1/2 from BDNFstimulated neurons. Cortical neurons were treated as indicated. Proteins immunoprecipitated with a KSR1 antibody were analyzed by Western blotting for KSR1, active ERK1/2 (phosphorylated at Thr ${ }^{183}$ and Tyr ${ }^{185}$, pERK1/2), total ERK1/2 (ERK1/2) or MKK1/2. BDNF induced the association between KSR1 and pERK1/2. Note the presence of total ERK1/2 associated with KSR1 in unstimulated neurons. No pERK1/2 or ERK1/2 was found if the immunoprecipitation (IP) was performed using nonimmune lgG (data not shown). The interactions between KSR1 and MKK1/2 were unaffected by BDNF. Similar levels of KSR1 indicated consistent immunoprecipitation. The graph presents quantitative analysis of pERK1/2-KSR1 association from three independent experiments. Error bars are SEM. $p<0.001$ (ANOVA). C, D, KSR1 colocalizes with pERK1/2 in BDNF-stimulated cultured rat cortical neurons. $C$, Immunofluorescence analysis of KSR1 and pERK1/2 at the indicated times after addition of $10 \mathrm{ng} / \mathrm{ml}$ BDNF. At 0, 2, or 5 min after BDNF addition, perikarial KSR1 immunoreactivity was concentrated in membrane-containing Golgi-like structures (arrows). BDNF increased pERK1/2 immunoreactivity at all time points. Z-stack confocal images are presented. D, Z-stack confocal images of representative neurons coimmunostained for KSR1 and pERK at 5 min after BDNF stimulation. Note the colocalization of pERK1/2 and KSR1 in membrane-containing structures with concentrated KSR1 immunostaining.

in PBS/0.3\% Triton X-100) were applied overnight at $4^{\circ} \mathrm{C}$. To enhance the signal for KSR1, biotinylated anti-rabbit antibody was added followed by the incubation with streptavidine-Alexa 555 conjugates. Antimouse Alexa 488 antibody was used to detect pERK. KSR1 and pERK1/2 images were acquired using Nikon (Tokyo, Japan) D-Eclipse C1 confocal microscope and processed using EZ-C1 software (Nikon).

Western blotting. Western blot analysis was performed using a standard procedure as suggested by Cell Signaling Technology. The dilutions of the primary antibodies were as follows: pERK1/2 (1:2000), ERK1/2 (1:1000), KSR1 (1:1000; donated by D. Morrison), pMKK1/2 (1:1000),
MKK1/2 (1:1000), Trk (1:1000), pTrkB (1:1000), pPKA-substrates (1:1000), flag (1:500), and $\beta$-actin (1:100).

Immunoprecipitation. Cells were washed with PBS, harvested in radioimmunoprecipitation assay buffer (50 mm Tris- $\mathrm{HCl}, \mathrm{pH} 7.4,150 \mathrm{~mm}$ $\mathrm{NaCl}, 0.5 \%$ deoxycholic acid, 1\% NP-40, $1 \mathrm{~mm}$ EDTA, 1 mM PMSF, 1 mm sodium orthovanadate, $1 \mathrm{~mm}$ sodium fluoride, $1 \mu \mathrm{g} / \mathrm{ml}$ aprotinin, $1 \mu \mathrm{g} / \mathrm{ml}$ leupeptin, $50 \mu \mathrm{l} / 10^{6}$ cells) and incubated for $30 \mathrm{~min}$ at $4^{\circ} \mathrm{C}$. The supernatants were collected after a 30 min microfuge centrifugation at $13,000 \mathrm{rpm}$. After preclearing with protein A-Sepharose, samples were incubated with $8-10 \mu \mathrm{g} /$ sample of mouse monoclonal antiKSR antibody (Upstate Biotechnology) at $4^{\circ} \mathrm{C}$ overnight. The immunocomplexes were precipitated with protein G-Sepharose beads (GE Healthcare, Piscataway, NJ) for $3 \mathrm{~h}$ at $4^{\circ} \mathrm{C}$ and analyzed by Western blotting. For immunoprecipitation of TrkB, we used a polyclonal antiTrk antibody (C-14, 1-2 $\mu \mathrm{g} / \mathrm{sample}$; Santa Cruz Biotechnology) and protein A-Sepharose beads (GE Healthcare).

Trophic withdrawal. Medium was removed from cultures and saved (serum-containing conditioned medium). Cells were washed twice with serum-free BME and incubated in serumfree BME supplemented with $35 \mu \mathrm{M}$ glucose, 1 $\mu \mathrm{M} \mathrm{L}$-glutamine, $100 \mathrm{U} / \mathrm{ml}$ penicillin, $0.1 \mathrm{mg} / \mathrm{ml}$ streptomycin, and a NMDAR blocker, MK-801 $(1 \mu \mathrm{M})$. Control cells were washed the same way and incubated in the serum-containing conditioned medium containing $1 \mu \mathrm{M}$ MK-801.

Quantitation of neuronal survival using MTT assay. The MTT assay was performed as described previously (Hetman et al., 1999).

Quantitation of apoptosis based on changes in nuclear morphology. To evaluate nuclear morphology, cells were stained with Hoechst 33258 and observed using fluorescent microscopy as described previously (Hetman et al., 1999). Cells with condensed or fragmented nuclei were scored as apoptotic. At least 300 cells were scored for each condition in each experiment. In experiments involving transfected neurons, apoptotic cell death was scored in $\beta$-galpositive cells. At least 150 cells were analyzed for each condition in each experiment.

Caspase activity. Caspase- 3 activity was determined using a colorimetric assay (Promega) as described previously (Gozdz et al., 2003).

Statistical analysis. Statistical analysis of the data was performed using one-way ANOVA followed by Bonferroni post hoc comparisons.

\section{Results}

\section{KSR1 expression in rat brain}

To test the role of KSR1 in survival signaling networks activated by BDNF, we decided to use cultured primary cortical neurons from newborn rats. In these cells, BDNF robustly activates ERK1/2 and suppresses apoptosis that is induced by DNA damage or TD (Hetman et al. 1999, 2002). Because brain expression of KSR1 has been studied in mice but not rats (Muller et al., 2000; Giblett et al., 2002), we performed immunostainings to verify KSR1 presence in rat cortical neurons. KSR1 immunoreactivity was present in forebrain including neocortex and hippocampus (Fig. 1A). It was 
localized to perikarial regions of neuron-like cells (Fig. $1 A c^{\prime}, e^{\prime}$ ). Frequent KSR1-positive cell bodies were observed throughout all cortical regions, and those cortical layers that have high representation of neuronal bodies including neocortical layers 4/5 (Fig. $\left.1 A c^{\prime}\right)$. Also, multiple KSR1-positive cells were present in these hippocampal areas that like stratum pyramidale of CA3 contained many neuronal cell bodies (Fig. $1 A e^{\prime}$ ). Overall, the pattern of KSR1 expression in rat forebrain is similar to that reported for mouse (Muller et al., 2000; Giblett et al., 2002).

\section{BDNF signaling involves KSR1 interactions with ERK1/2}

To test the role of KSR1 in BDNF-mediated activation of ERK1/2, cortical neurons were stimulated with BDNF and KSR1 was immunoprecipitated. Western blot analysis of the immunoprecipitate confirmed presence of KSR1 and demonstrated KSR1 interactions with the key proteins of the ERK1/2 pathway (Fig. $1 B)$. BDNF increased coimmunoprecipitation of KSR1 and pERK1/2 (Fig. 1B). However, the interaction between KSR1 and MKK1/2 appeared constitutively present and unaffected by BDNF (Fig. $1 B$ ). This is consistent with previous reports indicating that KSR1 associates inducibly with pERK1/2 and constitutively with MKK1/2 (Cacace et al., 1999; Muller et al., 2000).

To further characterize the interactions between $\mathrm{pERK} 1 / 2$ and KSR1 in BDNF-stimulated neurons, we performed indirect immunofluorescence analysis of KSR1 and pERK1/2. In unstimulated cells, KSR1 staining was found in perikaria (Fig. 1C). KSR1 appeared concentrated in membrane-like structure resembling Golgi apparatus (Fig. 1C, arrows). In addition, strongly positive nucleolar-like bodies were found in many neurons. Such structures were not present in staining controls with nonimmune IgG (data not shown). However, their occasional extraperikarial (possibly extracellular) occurrence indicated that they may represent a nonspecific staining. At 2 or $5 \mathrm{~min}$, BDNF did not appear to cause major redistribution of KSR1 in the cells (Fig. 1C). In unstimulated neurons, pERK staining was weak (Fig. 1C). BDNF strongly increased pERK1/2 signal. The increase was evident in perikarial cytosol (Fig. 1C). At $30 \mathrm{~min}$ after BDNF treatment, pERK was also visible in nuclei of many neurons (data not shown).

At 5 min after BDNF treatment, pERK1/2 and KSR1 colocalized in membrane-containing structures inside of neuronal perikaria (Fig. 1 D). Similar pattern was observed at 2 and $30 \mathrm{~min}$ (data not shown). Altogether, the BDNF-induced interactions of KSR1 with pERK1/2 suggest a role for KSR1 in activation of the cortical neuron ERK1/2 pathway after neurotrophic stimulation.

\section{KSR1 contributes to BDNF-mediated activation of ERK1/2}

To test the role of KSR1 in ERK1/2-mediated responses to BDNF, we developed three KSR1-targeting shRNAs. To validate these shRNAs, we electroporated cortical neurons with expression vectors for $\beta$-gal and murine WT-KSR1 together with each of the shKSR1 constructs. An shRNA plasmid targeting GFP was used as a control. The shKSR1 plasmids reduced expression of WT-KSR1 as determined by Western blot analysis $48 \mathrm{~h}$ after electroporation (Fig. 2A). None of the shRNAs affected the expression levels of $\beta$-gal (Fig. 2A). Thus, shKSR1 plasmids specifically inhibited WT-KSR1 expression.

Next, we tested whether KSR1 is required for BDNF-mediated activation of ERK1/2. Cortical neurons were electroporated with a mix of three shKSR1 plasmids (shKSR1) or shGFP together with flag-Bcl-2 expression plasmid. After $48 \mathrm{~h}$, neurons were stimulated with $10 \mathrm{ng} / \mathrm{ml} \mathrm{BDNF}$ for 10 or $30 \mathrm{~min}$. Neurons receiving shKSR1 had reduced increases of pERK1/2 levels compared with
A

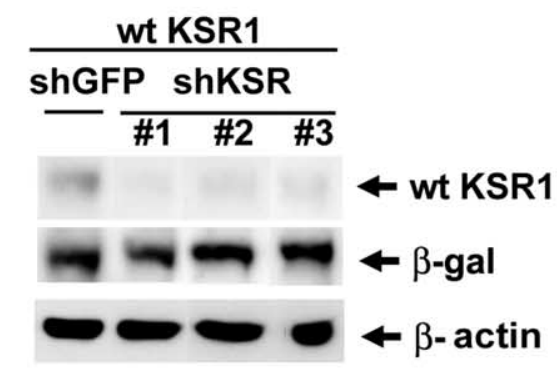

B
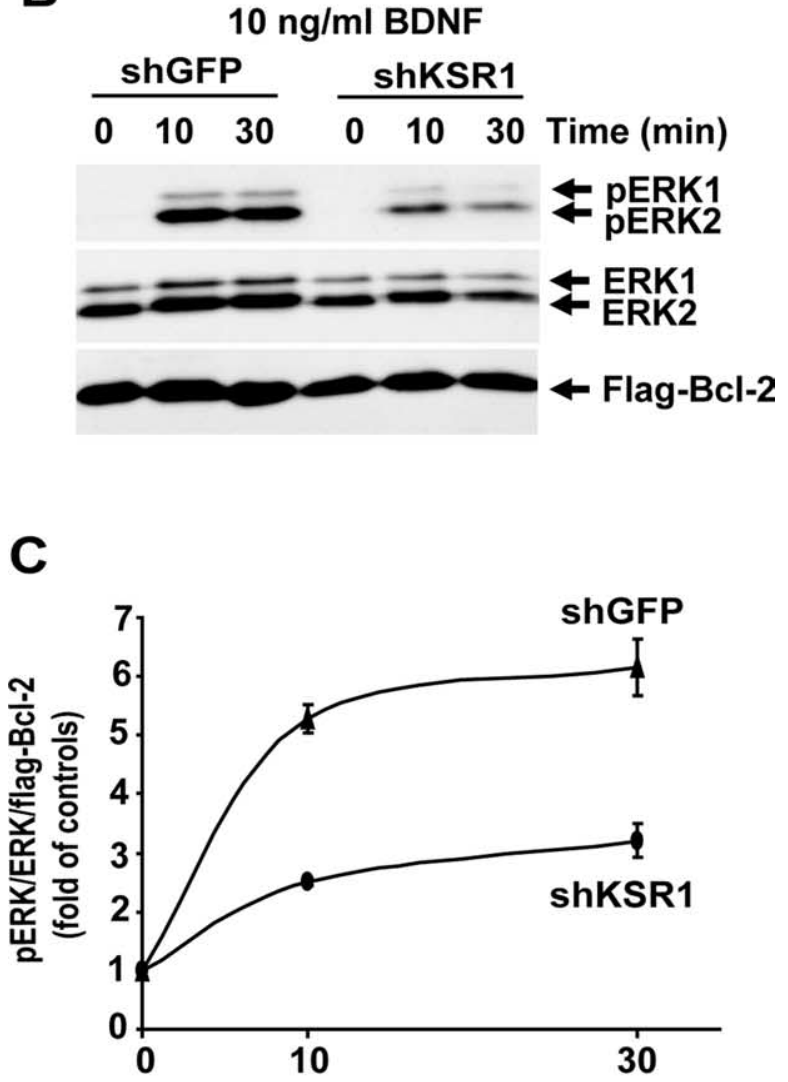

Time with 10ng/ml BDNF (min)

Figure 2. KSR1 contributes to BDNF-mediated activation of ERK1/2. A, Validation of shRNA constructs targeting KSR1. Freshly isolated neurons were coelectroporated with WT-KSR1 and $\beta$-gal expression constructs ( $1 \mu \mathrm{g}$ of wtKSR1 and $0.2 \mu \mathrm{g}$ of pON260 plasmid DNAs $/ 2 \times 10^{6}$ cells, respectively) together with $1.5 \mu \mathrm{g}$ of plasmid DNA $/ 2 \times 10^{6}$ cells of each of the following plasmids: control shRNA (shGFP) or one of three KSR1-specific shRNAs cloned in pSUPER as indicated (shKSR1 \#1, \#2, or \#3; see Materials and Methods for details). Forty-eight hours after electroporation, KSR1 levels were determined by Western blotting followed by reprobing the blot with an anti- $\beta$-gal or anti- $\beta$-actin antibodies to monitor transfection efficiency or protein loading, respectively. Each of the shKSR1 constructs inhibited WT-KSR1 expression. Similar results were obtained in another independent experiment. $\boldsymbol{B}$, Freshly isolated cortical neurons were coelectroporated with control shRNA (shGFP) or shKSR (a 1:1:1 mix of shKSR1 \#1, \#2, and \#3) together with a Flag-Bclll expression vector ( $4 \mu \mathrm{g}$ of shRNA and $0.5 \mu \mathrm{g}$ of pcDNA3-Flag$B C / 1 / 5 \times 10^{6}$ neurons, respectively). Two days later, neurons were stimulated with BDNF as indicated. The BDNF-mediated ERK1/2 activation was analyzed by Western blotting for pERK $1 / 2$ followed by reprobing for ERK $1 / 2$. In addition, reprobing for flag-BCl-2 was performed to monitor transfection efficiency. C, Analysis of pERK/ERK/Flag-Bcl-2 indicated that shRNAmediated knockdown of KSR1 reduced ERK1/2 activation in BDNF-stimulated neurons. Data from two independent experiments are presented. Error bars indicate SEM. 
A

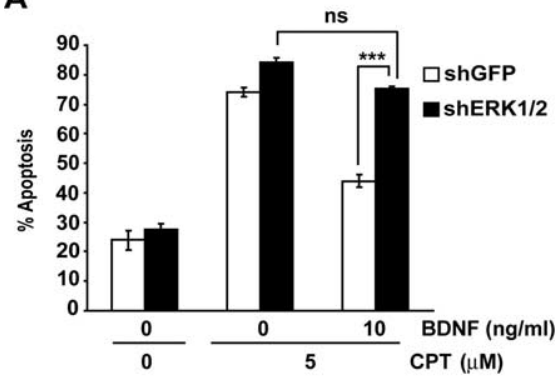

C

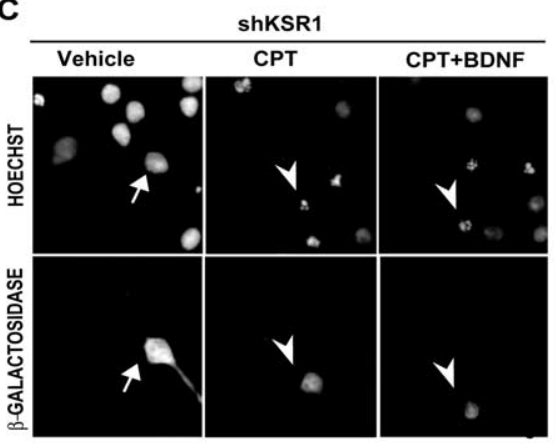

E

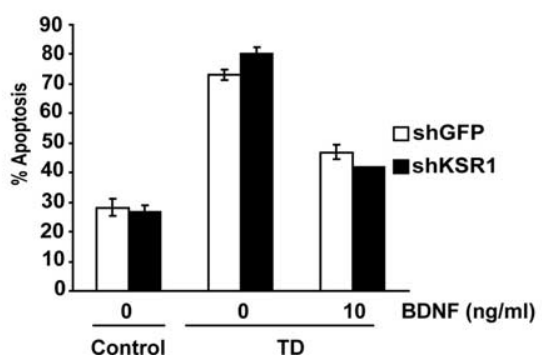

Figure 3. KSR1 is required for the BDNF-mediated protection against DNA damage. Cortical neurons were cotransfected with expression plasmids for $\beta$-gal (p0N260) and shKSR1 or shERK1/2 as indicated ( 0.2 and $1 \mu \mathrm{g}$ of plasmid DNAs/500,000 neurons, respectively); shGFP was used as a control. One day $(\boldsymbol{A}, \boldsymbol{B})$ or $3 \mathrm{~d}(\boldsymbol{D}, \boldsymbol{E})$ after transfection, neurons were exposed to $5 \mu \mathrm{M}$ (PT or TD for $24 \mathrm{~h}$. In the TD experiment, control neurons were subjected to sham washes (Control; see Materials and Methods for details). The treatments were performed in the presence or absence of $10 \mathrm{ng} / \mathrm{ml} \mathrm{BDNF}$. Transfected cells were identified by $\beta$-gal immunostaining. The morphology of the nuclei was visualized by counterstaining with Hoechst 33258 (HOECHST). $\boldsymbol{A}, \boldsymbol{B}$, Effects of shERK1/2 on BDNF neuroprotection against CPT- or TD-induced apoptosis. BDNF-mediated protection against CPT and TD was antagonized by shERK1/2. C, Representative photomicrographs depicting neurons transfected with shKSR1. Arrows indicate viable neurons; arrowheads indicate neurons with nuclear alterations indicating apoptosis (nuclear shrinkage and condensation with or without fragmentation). $\boldsymbol{D}, \boldsymbol{E}$, Effects of shKSR1 on BDNF neuroprotection against (PT- or TD-induced apoptosis. BDNFmediated protection against CPT but not TD was reduced by shKSR1. $F, K S R 1$ appears to be specific for the antiapoptotic activation of ERK1/2 that protects against CPT but not TD. In $\boldsymbol{A}, \boldsymbol{B}$, and $\boldsymbol{D}$, data represent averages of duplicate determinations from three independent experiments. In $\boldsymbol{E}$, averages of duplicate determinations from two independent experiments are shown. Error bars indicate SEM. ${ }^{*} p<0.05 ;{ }^{* *} p<0.01 ;{ }^{* * *} p<0.001$. ns, Nonsignificant.

shGFP-transfected cells ( 45 or $51 \%$ of pERK1/2 responses to 10 or 30 min BDNF treatments, respectively) (Fig. $2 B, C$ ). These data suggest KSR1 contribution to BDNF-triggered activation of ERK1/2 signaling. Therefore, KSR1 may be involved in ERK1/2mediated survival responses to BDNF.

\section{KSR1 contributes to antiapoptotic effects of BDNF}

We previously demonstrated that in cortical neurons, which were treated with DNA-damaging drugs including CPT, BDNF suppresses apoptosis by activating the ERK1/2 pathway (Hetman et al., 1999; Gozdz et al., 2003). Others have shown that ERK1/2 mediates BDNF protection against TD (Bonni et al., 1999). We confirmed these requirements of ERK1/2 by demonstrating that ERK1/2 knockdown blocked BDNF protection against CPT or
TD. For ERK1/2 knockdown, we used a mix of validated ERK1-1 and ERK2-1 shRNAs targeting rat ERKs. The validation of these constructs is presented in another manuscript (Kharebava, Kalita, and Hetman, unpublished observations). Neurons were cotransfected with shERK1/2 or shGFP used as a control. In addition, a $\beta$-gal expression plasmid was added to provide a marker of transfection. After $24 \mathrm{~h}$, cells were treated for next $24 \mathrm{~h}$ with $5 \mu \mathrm{M}$ CPT or TD that consisted of placement in serum-free medium supplemented with an NMDAR antagonist, MK801. Transfected neurons were identified by the presence of $\beta$-gal immunofluorescence, whereas apoptotic alterations of nuclear chromatin were visualized by Hoechst33258 counterstaining. In shGFPtransfected neurons that were treated with CPT, apoptosis was 75 or $44 \%$ in the presence of 0 or $10 \mathrm{ng} / \mathrm{ml}$ BDNF, respectively (Fig. 3A). In shERK1/2-transfected neurons, BDNF did not suppress CPT-induced apoptosis ( 84 or $75 \%$ apoptosis with 0 or 10 ng/ml BDNF, respectively; $p>0.05$ ) (Fig. $3 A$ ). A similar block of BDNF protection was found in shERK1/2-transfected neurons that were induced to die by TD (Fig. $3 B$ ). Finally, we obtained almost identical results using a shERK1/2 mix that contained plasmids targeting different regions of rat ERK1 and ERK2 (data not shown). Therefore, in cortical neurons, ERK1/2 is critical for BDNF suppression of either CPT- or TDinduced neuronal apoptosis.

To test the role of KSR1 in BDNF neuroprotection against $\mathrm{CPT}$ or $\mathrm{TD}$, shKSR1 was cotransfected with an expression vector for $\beta$-gal. The shGFP plasmid was used as a control for shKSR1. Three days after transfection, cells were exposed for $24 \mathrm{~h}$ to $5 \mu \mathrm{M}$ CPT in the presence or absence of 10 $\mathrm{ng} / \mathrm{ml}$ BDNF. Transfected neurons were identified by the presence of $\beta$-gal immunofluorescence and counterstained with Hoechst 33258 (Fig. 3C). Apoptosis in these cells was indicated by morphological alterations in nuclear chromatin including condensation and shrinkage with or without fragmentation (Fig. 3D). In shGFP-transfected neurons, there was 29\% apoptosis under basal conditions and 79\% after CPT treatment (Fig. 3D). Similar trends were observed in siKSR1-transfected neurons (Fig. 3D), suggesting that KSR1 knockdown does not affect neuronal responses to CPT. However, shKSR1 reduced the BDNF protection against CPT-induced apoptosis (38\% apoptosis in shGFP vs $60 \%$ apoptosis in shKSR1transfected neurons after CPT plus BDNF treatment; $p<0.01$ ) (Fig. 3D). Importantly, in shKSR1-transfected neurons, the apoptotic response to CPT was not significantly affected by BDNF (72\% apoptosis after CPT vs $60 \%$ after CPT plus BDNF treatment) (Fig. 3D), indicating removal of BDNF protection in the absence of KSR1. In contrast, the protective activity of BDNF against TD-induced apoptosis was unaffected by shKSR1 (Fig. 
$3 E)$. These results suggest that KSR1 is required for ERK1/2 activation that interferes with apoptotic cascades induced by DNA damage but not TD (Fig. $3 F$ ).

The extent of BDNF-mediated suppression of CPT-induced apoptosis has been shown before to depend on BDNF concentration. Also, overexpression of KSR1 enhanced responses to neurotrophic stimulation (Hetman et al., 1999). Therefore, we tested whether overexpressed KSR1 can increase protection against CPT-induced apoptosis by suboptimal concentrations of BDNF. Neurons were transfected with WT-KSR1 together with a $\beta$-gal expression plasmid. After $48 \mathrm{~h}$, neurons were treated with $5 \mu \mathrm{M}$ CPT in the presence of 0 or $10 \mathrm{ng} / \mathrm{ml} \mathrm{BDNF}$. After the next $24 \mathrm{~h}$, CPT induced 63 or $64 \%$ apoptosis in neurons receiving empty vector or WT-KSR1, respectively (Fig. 4). Therefore, overexpression of WT-KSR1 was not sufficient to reduce CPT-induced apoptosis. In contrast, cells treated with CPT and $1 \mathrm{ng} / \mathrm{ml}$ BDNF demonstrated 45 or $23 \%$ apoptosis after receiving empty vector or WT-KSR1, respectively (Fig. 4). However, the more efficient protection by $10 \mathrm{ng} / \mathrm{ml}$ BDNF ( 63 or $26 \%$ apoptosis in empty vector-transfected neurons with 0 or $10 \mathrm{ng} / \mathrm{ml} \mathrm{BDNF}$, respectively) was unaffected by WT-KSR1 (Fig. 4). Therefore, overexpression of WTKSR1 augmented the ability of a subprotective BDNF concentration to suppress CPT-induced apoptosis.

\section{Role of KSR1 in cAMP-mediated activation of neuronal ERK1/2}

Our results demonstrate that KSR1 plays a role in the ERK1/2-mediated suppression of DNA damage-induced apoptosis. An important issue raised by these observations is that of generality of KSR1 involvement in ERK1/2 signaling. For instance, one can speculate that KSR1 may be required for the antiapoptotic activation of ERK1/2 by other stimuli than BDNF. Increased cAMP levels have been shown to activate antiapoptotic signaling by ERK1/2 in cerebellar granule and retinal ganglion neurons (Villalba and Journot, 1997; Meyer-Franke et al., 1998). Therefore, we tested whether elevated cAMP levels can produce KSR1- and ERK1/2mediated protection against DNA damage-induced apoptosis in cortical neurons. We first verified that in cortical neurons cAMP activates ERK1/2. A cAMP-elevating drug, forskolin $(10 \mu \mathrm{M})$, increased levels of phosphorylated MKK1/2 and ERK1/2 (pMKK1/2 and pERK1/2, respectively), indicating activation of the ERK1/2 pathway (Fig. $5 A$ ). The levels of pMKK1/2 and pERK1/2 increased as early as $30 \mathrm{~min}$ and remained elevated for at least $4 \mathrm{~h}$ after the treatment (Fig. $5 \mathrm{~A}$ ). The forskolin-induced increase in pERK1/2 was abolished with the MKK1/2 inhibitor U0126, suggesting MKK1/2 involvement in cAMP effects on ERK1/2 (Fig. $5 A$ ).

BDNF, but not forskolin, activation of ERK1/2 was accompanied by stimulation of TrkB, indicating that in cortical neurons the signaling between cAMP and ERK1/2 was independent of BDNF/TrkB (Fig. 5B). Instead, it involved PKA because PKA inhibition using either the pharmacological inhibitor H89 or a mediated by different pathways.

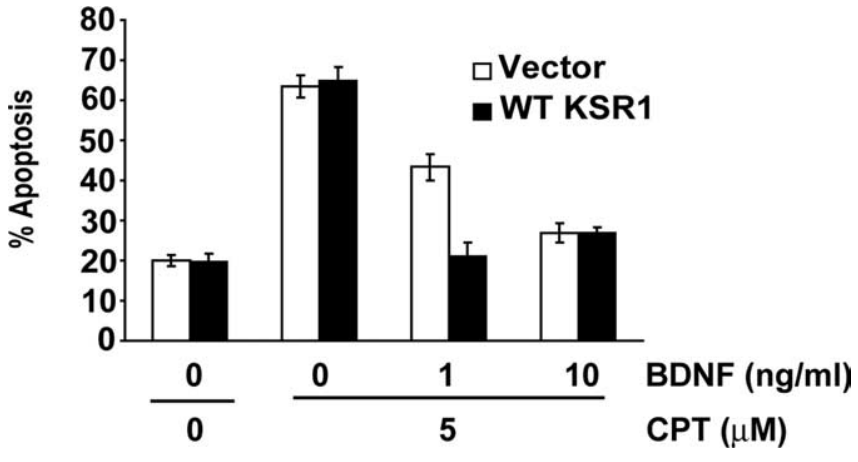

Figure 4. Overexpression of wtKSR1 increases BDNF protection against CPT-induced apoptosis. Cortical neurons were cotransfected with expression plasmids for $\beta$-gal and WT-KSR1 or an empty cloning vector, pcDNA3.1 (0.2 and $0.03 \mu \mathrm{g}$ of plasmid DNAs $/ 5 \times 10^{5}$ neurons, respectively). Two days after transfection, neurons were exposed to $5 \mu \mathrm{M}$ (PT in the presence of 0 or 1 or $10 \mathrm{ng} / \mathrm{ml}$ BDNF. After $24 \mathrm{~h}$, WT-KSR1 increased antiapoptotic protection by low but not high concentration of BDNF. Data are averages of duplicate determinations \pm SEM from two independent experiments.
A

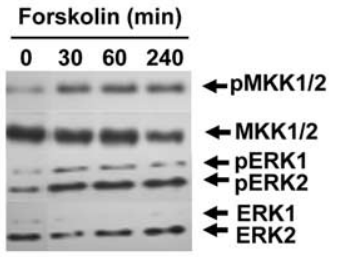

C

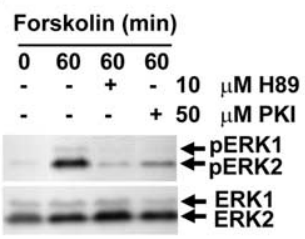

B
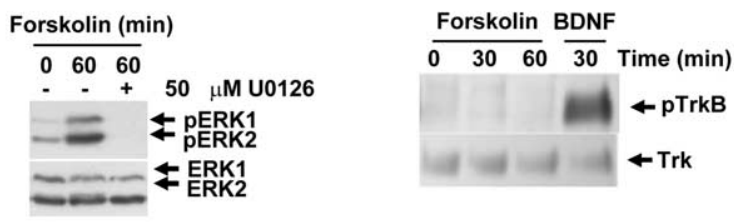

E

D

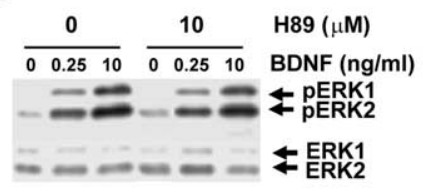

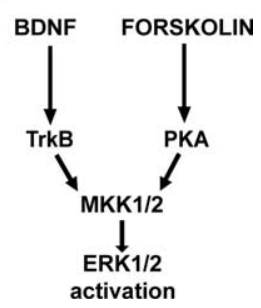

Figure 5. Stimulation of CAMP signaling activates ERK1/2 in a TrkB-independent and PKA-dependent manner. Cortical neurons were treated with the adenylyl cyclase activator forskolin $(10 \mu \mathrm{m})$ or BDNF as indicated. The levels of active (phosphorylated) forms of ERK1/2 (pERK1/2; $\boldsymbol{A}, \boldsymbol{C}, \boldsymbol{D})$ or MKK1/2 (pMKK1/2; $\boldsymbol{A}$ ) were analyzed by Western blotting. Levels of total ERK1/2 or MKK1/2 were determined by reprobing blots with appropriate antibodies. In $\boldsymbol{B}$, the level of activated TrkB that was phosphorylated at the phospholipase $\mathrm{C}-\gamma$ binding site (Y816, pTrkB) was determined by Western blotting after immunoprecipitation of total TrkB with a pan-Trk antibody. Total levels of Trk were revealed by reprobing with the pan-Trk antibody. All results were replicated in two to three independent experiments. $\boldsymbol{A}$, Forskolin activated MKK1/2 and ERK1/2. MKK1/2 inhibition with U0126 abolished ERK1/2 activation by forskolin, indicating a critical role of MKK1/2. $B$, ERK1/2 activation by forskolin was not accompanied by TrkB activation, suggesting that, in cortical neurons, TrkB is not involved in CAMP signaling to ERK1/2. In contrast, TrkB was strongly activated by $10 \mathrm{ng} / \mathrm{ml}$ BDNF. C, Forskolin failed to activate ERK1/2 in the presence of the PKA inhibitor H89 or myrPKI (PKI). D, BDNF-induced activation of ERK1/2 was not affected by PKA inhibition using $\mathrm{H} 89$. Effects of $\mathrm{H} 89$ were tested using both low and high concentrations of BDNF that were added for 30 min as indicated. $\boldsymbol{E}$, ERK1/2 activation in response to cAMP and BDNF is

membrane-permeable peptide inhibitor, myrPKI, greatly reduced forskolin activation of ERK1/2 (Fig. 5C). The PKA involvement was specific for cAMP- but not BDNF-mediated activation of ERK1/2 (Fig. 5D). Therefore, in cortical neurons, cAMP activated ERK1/2 via a PKA- but not TrkB-dependent signaling pathway (Fig. 5E). This differs from BDNF signaling to ERK1/2 that involved TrkB but not PKA (Fig. $5 E$ ).

To test whether in cortical neurons KSR1 is involved in cAMP activation of ERK1/2, we analyzed the KSR1 association with pERK1/2 using a coimmunoprecipitation assay. The KSR1- 
A

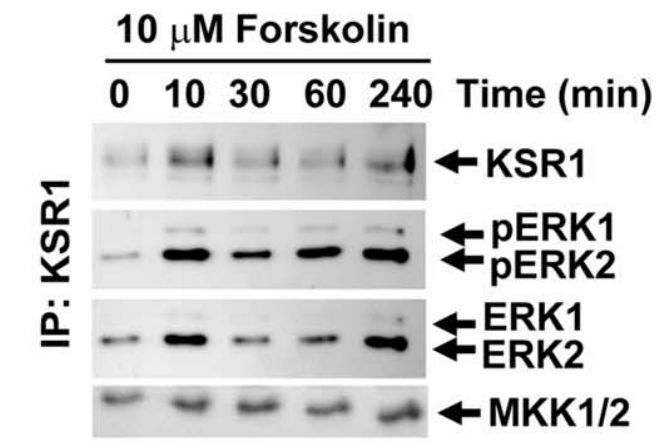

B

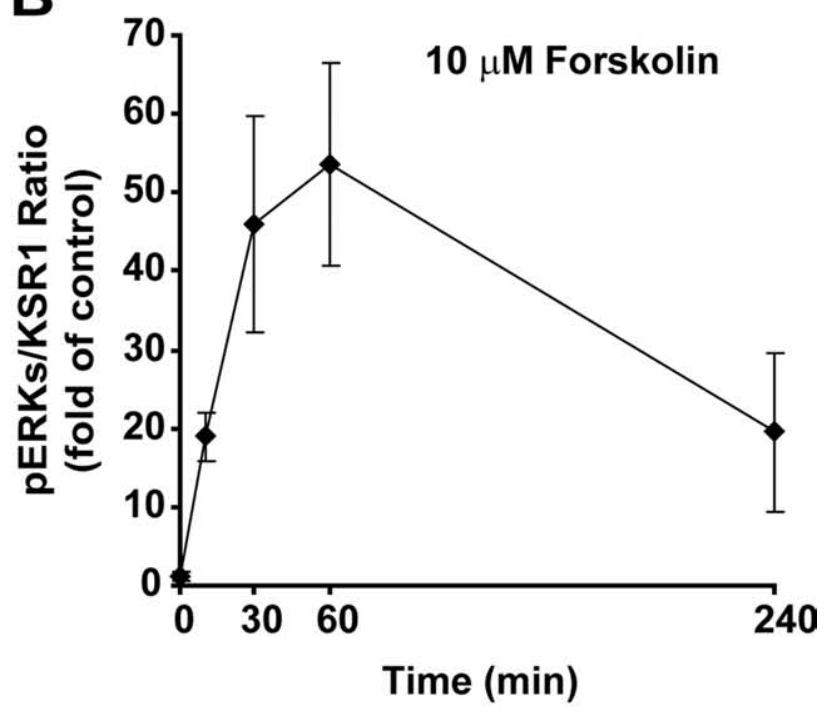

C

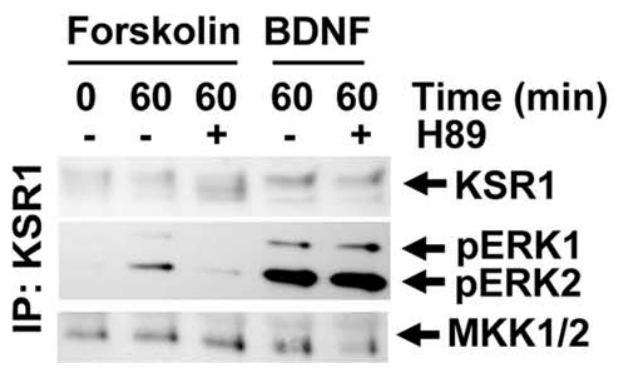

Figure 6. KSR1 associates with active ERK1/2 after stimulation of CAMP signaling. Neurons were treated as indicated. The association between phosphorylated ERK1/2 (pERK1/2) and KSR1 was studied by coimmunoprecipitation (IP) as described for Figure 1.A, Activation of CAMP signaling by forskolin increased the association between pERK1/2 and KSR1 without affecting MKK1/2 binding to KSR1. $\boldsymbol{B}$, Increased ratios between coimmunoprecipitated $\mathrm{pERK} 1 / 2$ and KSR1 from forskolin-stimulated neurons. Data in the graph represent three independent experiments. Error bars indicate SEM. $p<0.001$ (ANOVA). C, Inhibition of PKA with H89 (10 $\mu \mathrm{m})$ disrupted the forskolin $(10 \mu \mathrm{M})$-induced increase in KSR1-pERK1/2 association. $\mathrm{H} 89$ did not affect BDNF (10 $\mathrm{ng} / \mathrm{ml}$ )-induced interactions between KSR1 and pERK1/2. These results suggest a role for PKA in CAMP-mediated activation of KSR1/ERK signaling. Similar results were obtained in two independent experiments.

pERK1/2 interactions were increased as early as $10 \mathrm{~min}$ after treatment with $10 \mu \mathrm{M}$ forskolin (Fig. 6A,B). The maximum of that was detected at $60 \mathrm{~min}$ with strong association still present at least up to $4 \mathrm{~h}$ after treatment (Fig. 6B). The PKA inhibitor H89 abolished forskolin-induced interactions between KSR1 and pERK1/2 (Fig. 6C). H89 had no effect on the BDNF-induced association of KSR1 with pERK1/2 (Fig. 6C). Therefore, in cultured cortical neurons, KSR1 contributed to the cAMP/PKAmediated activation of ERK1/2.

We attempted to study the colocalization of pERK1/2 and KSR1 in forskolin-stimulated neurons as it was done for BDNF (Fig. 1C,D). However, the relatively weaker activation of ERK by forskolin was undectable by immucytochemistry (data not shown). As in the case of BDNF, we did not observe KSR1 redistribution in forskolin-stimulated neurons (data not shown). Unlike BDNF, forskolin did not activate ERK1/2 at DIV2 (Fig. $2 B, C$ and data not shown). However, at longer times after DIV0 electroporations, shRNA constructs induced nonspecific toxicity. Therefore, we were unable to assess the effects of shKSR1 on ERK1/2 activation in forskolin-stimulated neurons. Nevertheless, increased association of KSR1 and pERK1/2 indicates that, like BDNF, forskolin activates the KSR1-ERK1/2 signaling pathway.

\section{Critical role of KSR1 in neuroprotection by}

\section{cAMP-activated ERK1/2}

BDNF-activated signaling by KSR1-ERK1/2 protected against the DNA-damaging drug CPT (Fig. 3). Therefore, the KSR1ERK1/2 pathway that is activated by cAMP may have similar neuroprotective effects. To test this possibility, we evaluated whether activation of cAMP signaling by forskolin would reduce CPT-induced neuronal apoptotsis. In neurons that were treated for $48 \mathrm{~h}$ with $5 \mu \mathrm{M} \mathrm{CPT}, 10 \mu \mathrm{M}$ forskolin increased survival from 36 to $82 \%$ of controls (Fig. $7 A$ ). Likewise, the fraction of neurons with apoptotic morphology that increased after a $24 \mathrm{~h}$ CPT exposure was reduced by forskolin (Fig. $7 B$ ). In concert with these findings, CPT-induced activation of the apoptotic protease caspase 3 was suppressed by cotreatment with forskolin (Fig. 7C). Thus, activation of cAMP signaling by forskolin protects cortical neurons against DNA damage-induced apoptosis.

To determine whether cAMP protection is mediated by the ERK1/2 pathway, we used a pharmacological inhibitor of the ERK1/2 pathway, U0126. We also used an overexpressed wildtype form of the ERK1/2-specific phosphatase MKP3, which inhibits ERK1/2 signaling by removing the activating phosphorylations from ERK1/2. Either of these approaches abolished neuroprotection by forskolin (Fig. $7 D, E$ ). For instance, $24 \mathrm{~h}$ treatment with $\mathrm{CPT}$ induced $63 \%$ apoptosis, and forskolin reduced that to $23 \%(p<0.001)$ (Fig. $7 D)$. In the presence of U0126, CPT induced 76 or $67 \%$ apoptosis with or without forskolin, respectively, indicating a complete block of forskolin neuroprotection if the ERK1/2 pathway was inactive (CPT plus U0126 vs CPT plus U0126 plus forskolin, nonsignificant) (Fig. $7 D)$. Similar trends were observed in experiments with overexpressed MKP3 (Fig. 7E). Therefore, ERK1/2 is required for cAMP protection against DNA damage-induced apoptosis of cortical neurons.

To evaluate the role of KSR1 in cAMP-mediated suppression of apoptosis, we studied the effects of KSR1 inhibition on forskolin neuroprotection of CPT-treated neurons. Neurons were transfected with shKSR1 or shGFP as described for Figure 3. CPT (5 $\mu \mathrm{M})$ induced 79 or $74 \%$ apoptosis in shGFP- or shKSR1-transfected neurons, respectively (Fig. 8, nonsignificant). Forskolin $(10 \mu \mathrm{M})$ reduced CPT-induced apoptosis to $37 \%$ in shGFP-transfected neurons $(p<0.001)$ (Fig. 8). The protection was partially removed by shKSR1 with apoptosis at $57 \%$ after the CPT plus forskolin treatment (shGFP vs shKSR1, $p<0.001$ ) (Fig. 8). These results indicate 
that KSR1 contributes to neuroprotective activation of ERK1/2 by cAMP.

\section{Role of PKA in the neuroprotective} activation of KSR1-ERK1/2 by cAMP Our data presented in Figures 5, $C$ and $D$, and $6 C$ indicate that PKA activity is required for cAMP but not BDNF signaling to KSR1ERK1/2 in cortical neurons. In addition, results shown in Figs. 7 and 8 suggest a critical role of KSR1-ERK1/2 in cAMP protection of CPT-treated neurons. Therefore, one could hypothesize that PKA inhibition would antagonize cAMP but not BDNF neuroprotection against CPT. To verify that prediction, we tested the effects of PKA inhibitors on forskolin-mediated suppression of CPT-induced apoptosis. Thus, 57\% neurons were apoptotic after a $24 \mathrm{~h}$ exposure to $5 \mu \mathrm{M} \mathrm{CPT}$, although 27\% apoptosis was found if CPT treatment was in the presence of $10 \mu \mathrm{M}$ forskolin (Fig. 9A) ( $p<0.001)$. The pharmacological PKA inhibitor H89 completely blocked the forskolin protection against CPT by increasing apoptosis to $70 \%$ (CPT plus forskolin vs CPT plus forskolin plus H98, $p<0.001$; CPT vs CPT plus forskolin plus H98, nonsignificant) (Fig. 9A). H89 did not affect BDNF neuroprotection against CPT-induced apoptosis (data not shown). Removal of forskolin- but not BDNF-mediated neuroprotection was also observed in neurons that were transfected with an expression plasmid for the specific PKA protein inhibitor, PKI $\alpha$ (Fig. 9B,C). These results suggest that PKA is required for the antiapoptotic activation of KSR1ERK $1 / 2$ by cAMP.

cAMP activates both PKA-dependent and PKA-independent pathways (Enserink et al., 2002). Therefore, cAMP-activated PKA may or may not be sufficient to turn on the antiapoptotic signaling by KSR1ERK1/2. To test the sufficiency of PKA for cAMP-mediated protection, we used the selective activator of PKA, Sp. In neurons treated for $48 \mathrm{~h}$ with $5 \mu \mathrm{M}$ CPT, $100 \mu \mathrm{M} \mathrm{Sp}$ increased survival from 28 to $74 \%$ of controls (Fig. 10A). Activation of PKA by Sp was verified by demonstration of increased levels of phosphorylated PKA substrates after a 60 min treatment with $100 \mu \mathrm{M}$ Sp (Fig. 10B). Spmediated activation of PKA was accompanied by activation of ERK1/2 (Fig. $10 B$ ). Effects of Sp on PKA or ERK1/2 were blocked by the PKA inhibitor H89, whereas the MKK1/2 inhibitor U0126 blocked activation of ERK1/2 but not PKA (Fig. 10B). In addition, Sp induced a PKA-dependent association between KSR1 and pERK1/2 (Fig. 10C). These results indicate that selective activation of PKA is sufficient to produce protection against CPT-induced neuronal death and to activate KSR1-ERK1/2 signaling.

To test the role of KSR1-ERK1/2 in PKA-mediated neuroprotection, we treated neurons with $5 \mu \mathrm{M}$ CPT or $5 \mu \mathrm{M}$ CPT plus $100 \mu \mathrm{M} \mathrm{Sp}$ in the presence or absence of the ERK1/2 pathway inhibitor U0126 or shKSR1. Sp lowered apoptosis induced by a $24 \mathrm{~h}$ treatment with
B
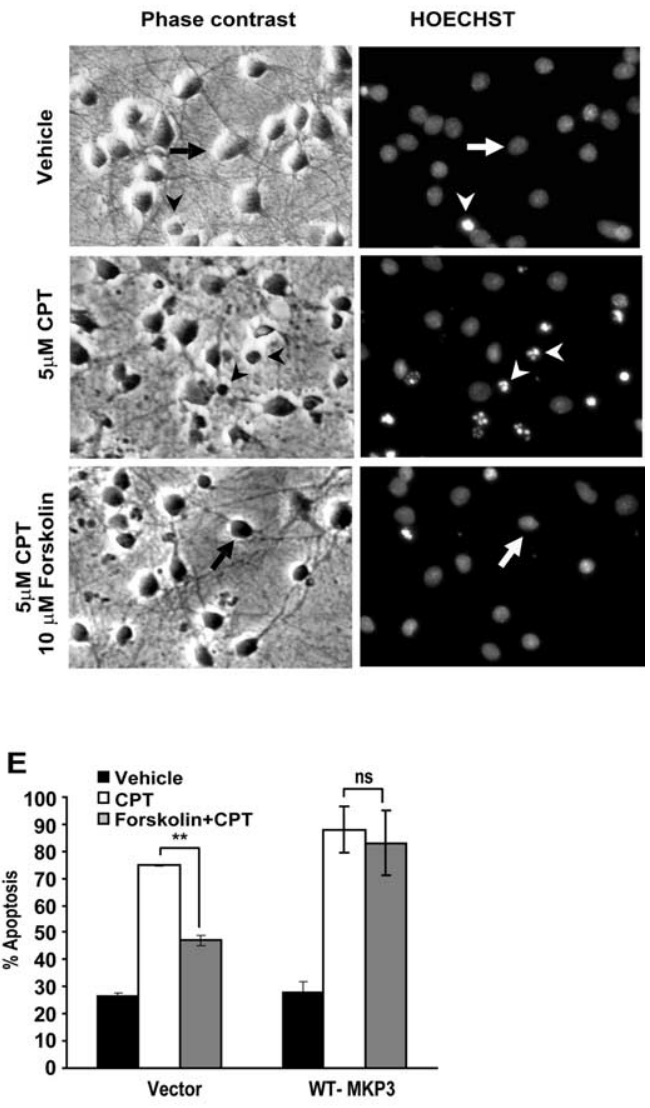

Figure 7. Stimulation of CAMP signaling inhibits DNA damage-induced apoptosis in an ERK1/2-dependent manner. $\boldsymbol{A}$, Neurons were treated with $5 \mu \mathrm{m}$ CPT for $48 \mathrm{~h}$ in the presence or absence of $10 \mu \mathrm{m}$ forskolin (Forsk) or $0.2 \%$ DMSO (Veh). Neuronal survival SEM. Forskolin reduced (PT-induced neurotoxicity $\left({ }^{* *} p<0.01\right)$. $\boldsymbol{B}$, Morphology of neurons protected with forskolin against toxicity by a $24 \mathrm{~h}$ CPT exposure. Hoechst staining and corresponding phase-contrast images are presented. Arrows indicate viable ttenuated by forskolin. C, Forskolin reduced (PT-induced caspase-3 activity. Data are averages of triplicate determinations from

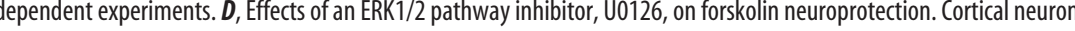
mediated-protection against CPT, indicating a critical role for the ERK1/2 pathway in the antiapoptotic effects of CAMP signaling in CPT-treated neurons. Data represent averages of duplicate determinations from four independent experiments \pm SEM. ${ }^{* *} p<$ 0.01. $\boldsymbol{E}$, Cortical neurons were cotransfected with expression plasmids for $\beta$-gal (p0N260) and an ERK1/2-specific phosphatase, MKP3 (WT-MKP3; 0.2 and $0.3 \mu \mathrm{g}$ of plasmid DNA/500,000 cells, respectively). The empty cloning vector pcDNA3.1 (Vector) was Quantification of apoptosis in transfected neurons indicated removal of forskolin-mediated protection by WT-MKP3. These data independent experiments \pm SEM. ${ }^{* *} p<0.01$. ns, Not significant.

CPT from 58 to $26 \%$ (Fig. 10D) $(p<0.001)$. With U0126, CPT induced $45 \%$ apoptosis despite the presence of Sp, indicating that ERK1/2 inhibition reduced PKA-mediated neuroprotection (CPT plus Sp vs CPT plus Sp plus U0126, $p<0.001$ ) (Fig. 10D). Similar trends were observed after inhibition of KSR1 with shKSR1 (Fig. $10 E$ ). Although CPT-induced apoptosis was effectively inhibited by Sp in shGFP-expressing neurons, no protection occurred after transfection of shKSR1 (CPT vs CPT plus Sp, $p<0.001$ for shGFPtransfected and $p>0.05$ for shKSR1-transfected neurons, respectively) (Fig. 10E). These data demonstrate that selective activation of PKA is sufficient to recruit the antiapoptotic signaling by KSR1ERK1/2. Thus, the antigenotoxic pathway that involves KSR1ERK1/2 converges with the protective signals delivered by BDNF or cAMP/PKA (Fig. 10F). 


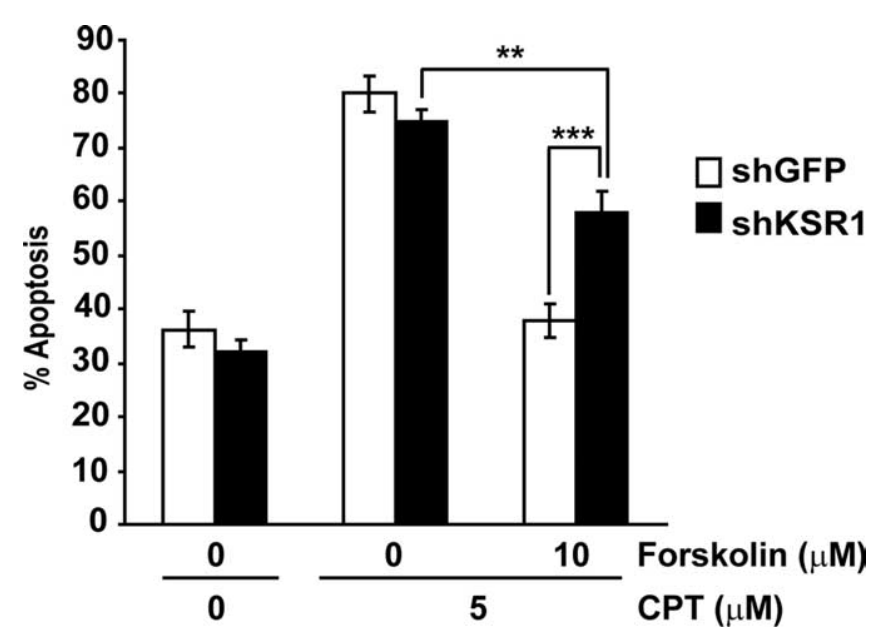

Figure 8. Role of KSR1 in CAMP neuroprotection against DNA damage. Neurons were transfected as in Figure $2 B-D$. Seventy-two hours after transfection, neurons were treated for $24 \mathrm{~h}$ as indicated. Quantification of apoptosis in transfected neurons revealed that shKSR1 reduced forskolin-mediated protection against CPT. Data represent averages of duplicate determinations from four independent experiments \pm SEM. ${ }^{* *} p<0.01 ;{ }^{* * *} p<0.001$.

\section{Discussion}

In this study, we demonstrated that in cortical neurons, KSR1 contributed to BDNF activation of ERK1/2 and to ERK1/2dependent BDNF responses, including suppression of DNA damage-induced apoptosis. We also showed that PKAdependent and TrkB-independent activation of ERK1/2 by cAMP involved KSR1 and provided antigenotoxic neuroprotection.

Presented results identified two new stimuli that activated neuronal KSR1-ERK1/2 signaling. BDNF or elevated levels of CAMP increased the association between the active ERK1/2 and KSR1 and induced neuronal responses that were both ERK1/2 and KSR1 dependent, including protection from DNA damageinduced apoptosis. It is well established that BDNF activates ERK1/2 by interactions with the TrkB receptor (Kaplan and Miller, 2000). In the case of cAMP signaling, our results indicate that TrkB was not activated, whereas PKA was required for turning on the ERK1/2 pathway. Thus, KSR1 contributes to ERK1/2 activation by two diverse signaling pathways: BDNF/TrkB and cAMP/PKA. The potential role of KSR1 in ERK1/2 activation by neurotrophins was indicated by experiments in PC12 cells that overexpressed KSR1 (Muller et al., 2000). However, contrary to our observation in cAMP-stimulated cortical neurons from newborn rats, hippocampal slice preparations from adult mice showed cAMP activation of ERK1/2 that was unaffected by KSR1 knock-out (Shalin et al., 2006). Similarly, there was no increase in association between pERK1/2 and KSR1 in cAMP-stimulated hippocampal slices from normal mice (Shalin et al., 2006). Finally, cAMP-dependent forms of long-term potentiation have not been disturbed by lack of KSR1 (Shalin et al., 2006). At present, one can speculate about possible reasons for the discrepancies between our results and those reported by Shalin et al. (2006). For instance, one possibility is that hippocampal neurons regulate KSR1-ERK1/2 differently than cortical neurons. Another possibility is that CAMP-mediated regulation of KSR1ERK1/2 is restricted to relatively young neurons such as those used in our experiments (cortical neurons from newborn rats). Neuronal survival in the developing nervous system is strongly dependent on the extracellular signals (Jacobson et al., 1997). Therefore, young neurons may benefit from having more inputs

\section{A}
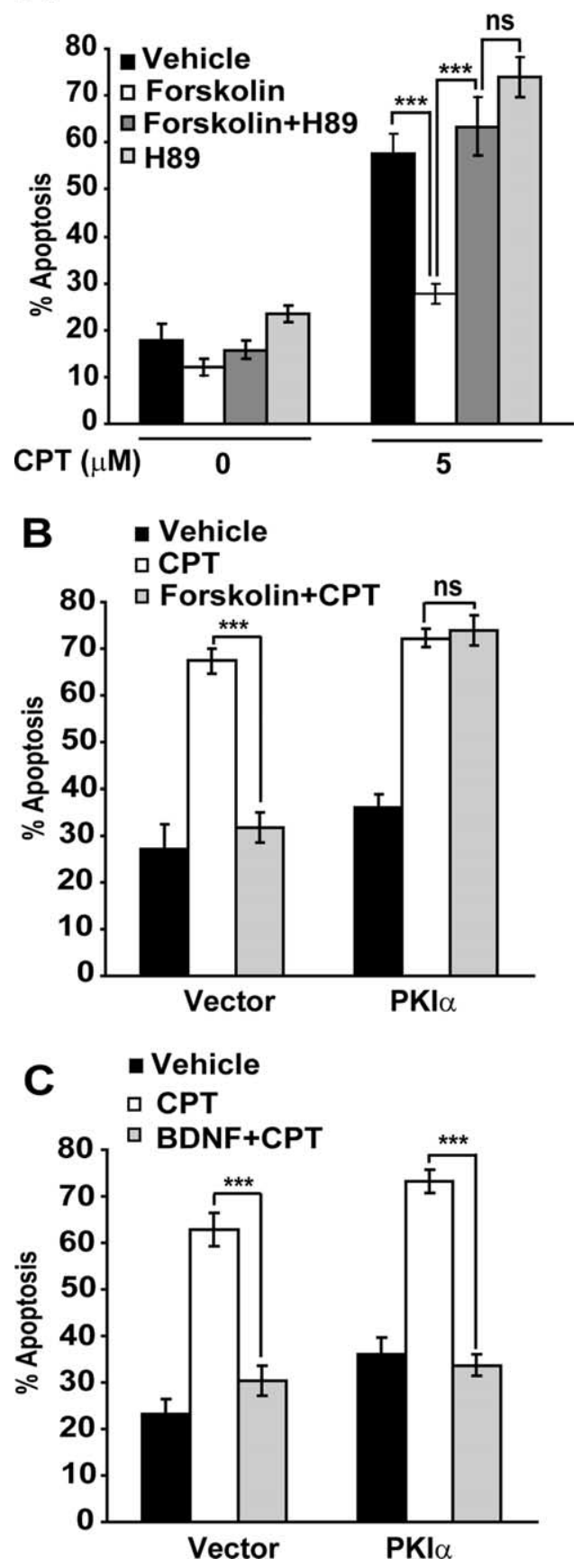

Figure 9. PKA is required for CAMP-mediated neuroprotection against CPT-induced apoptosis. $\boldsymbol{A}$, A $24 \mathrm{~h}$ CPT treatment of cortical neurons was performed in the presence or absence of $10 \mu \mathrm{m}$ forskolin and/or $10 \mu \mathrm{M}$ PKA inhibitor H89 as indicated. $\mathrm{H} 89$ abolished antiapoptotic effects of forskolin. $\boldsymbol{B}, \boldsymbol{C}$, Cortical neurons were cotransfected with expression constructs for $\beta$-gal (p0N260) and an endogenous protein inhibitor of PKA, PKI $\alpha$ ( 0.2 and $0.3 \mu \mathrm{g}$ of plasmid DNAs $/ 500,000$ neurons, respectively). Empty cloning vector (Vector, pcDNA3.1) was used as a control for PKl $\alpha$ construct. Twenty-four hours after transfection, neurons were treated with $5 \mu \mathrm{M}$ CPT in the presence or absence of $10 \mu \mathrm{m}$ forskolin or $10 \mathrm{ng} / \mathrm{ml} \mathrm{BDNF}$ as indicated. After $24 \mathrm{~h}$, apoptosis was analyzed in transfected neurons. Forskolin but not BDNF neuroprotection against CPT was abolished by the overexpressed PKl $\alpha$. Data in all panels represent averages of duplicate determinations from three independent experiments \pm SEM. ${ }^{* * *} p<0.001$. ns, Not significant.

activating prosurvival signaling by KSR1-ERK1/2. Finally, it is also possible that KSR1 does not mediate PKA activation of ERK1/2 serving, instead, as a downstream mediator of antiapoptotic PKA-ERK1/2 signaling in forskolin-stimulated neurons. 
Previous reports with overexpressed epitope-tagged KSR1 suggested that KSR1 undergoes dynamic redistribution to the plasma membrane after growth factor stimulation of Cos or NIH3T3 cells (Muller et al., 2001; Ory et al., 2003). In rat cortical neurons, we observed endogenous KSR1 localized to membrane-containing structures resembling the Golgi apparatus. This pattern did not appear to change after BDNF stimulation. Thus, in cultured neurons, endogenous KSR1 may be constitutively associated with the membranes. The possible presence of KSR1/pERK1/2 in the Golgi supports an interesting possibility that Golgi membranes are the site of ERK activation. Such a role has been proposed for this organelle based on the localization of MEK/ERK complexes with another scaffold protein, Sef (Torii et al., 2004). Subcellular distribution of endogenous KSR1 requires additional studies.

Yan et al. (2001) have implicated KSR1 in antiapoptotic signaling by tumor necrosis factor (TNF) in intestinal mucosa epithelia (Yan et al., 2001). In that system, KSR1 controlled the antiapoptotic activation of ERK1/2 and nuclear factor $\kappa$-B (NF $\kappa-\mathrm{B})$ (Yan et al., 2001). Although our results demonstrate neuroprotective interaction between KSR1 and ERK1/2, the role of KSR1 in neuronal survival by $\mathrm{NF} \kappa$-B remains to be studied. The role of ERK $1 / 2$ as a partner for KSR1 in mediating cell survival has been recently demonstrated in mouse embryonic fibroblasts (McKay and Morrison, 2007). In that system, apoptotic treatment with TNF induced caspase-mediated processing of KSR1 shutting down antiapoptotic ERK1/2 signaling (McKay and Morrison, 2007). Finally, the lack of KSR1 impaired TNF-mediated activation of Akt, indicating possible involvement in phosphatidylinositol 3'-kinase/Akt-mediated survival (Yan et al., 2004). In cortical neurons, we also found BDNFinduced KSR1 association with the activated form of Akt (E. Szatmari and Hetman, unpublished observations). Thus, the role of KSR1 in neuroprotective activation of Akt deserves additional studies.

We showed that KSR1-ERK1/2 signaling is a convergence point for antiapoptotic pathways that are activated by BDNF and cAMP. In the case of BDNF, KSR1 involvement was specific for antiapoptotic protection against the DNA-damaging drug CPT. Interestingly, also TD-induced apoptosis was suppressed by BDNF acting via the ERK1/2 pathway. Thus, similarly to cerebellar granule neurons placed in low $\mathrm{KCl}$, serum-free medium, cortical neuron apoptosis induced by serum deprivation in the presence of an NMDAR blocker is antagonized by BDNF in a ERK1/ 2-dependent manner (Bonni et al., 1999). However, KSR1 was not involved in BDNF-mediated protection against TD. This suggests specific requirement for KSR1-ERK1/2 in neuroprotective activity of BDNF in DNA-damaged neurons. Also, these results
B

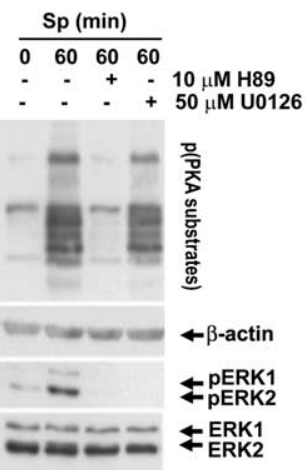

E

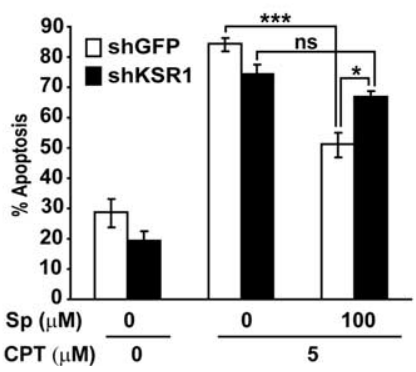

C

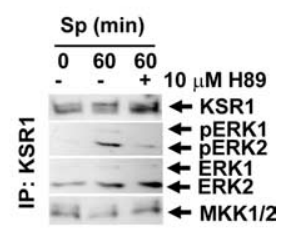

$\mathbf{F}$

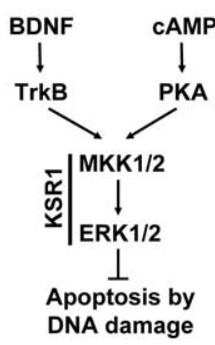

Figure 10. PKA is sufficient to activate protective signaling by KSR1-ERK1/2.A, Cortical neurons were treated with CPT for $48 \mathrm{~h}$ in the presence or absence of a selective PKA activator, Sp (100 $\mu \mathrm{M})$. Neuronal survival was determined by the MTT assay. PKA

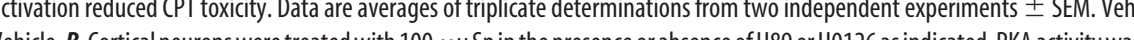
determined by Western blotting with an antibody specific for PKA substrate sites after PKA-mediated phosphorylation [p(PKA subtrates)]. To control for equal protein content, $\beta$-actin levels were determined by reprobing the blots. In addition, ERK1/2 activation was monitored by pERK1/2 levels. PKA activation was inhibited by $\mathrm{H} 89$ but not U0126. In contrast, Sp-induced ERK1/2 was blocked by either of these drugs. Hence, PKA is sufficient to activate ERK1/2. C, Cortical neurons were treated with described in Figure 1.Sp induced KSR1-pERK1/2 association, which was abolished by $\mathrm{H} 89$. Therefore PKA is sufficient to activate ERK1/2 through KSR1. Results presented in $\boldsymbol{B}$ and $\boldsymbol{C}$ were replicated in independent experiments. $\boldsymbol{D}$, Effects of an ERK1/2 pathway inhibitor, U0126, on Sp neuroprotection. Cortical neurons were treated for $24 \mathrm{~h}$ with CPT in the presence or absence of $100 \mu \mathrm{m} \mathrm{Sp}$ and/or $50 \mu \mathrm{m}$ U0126. U0126 reduced the Sp mediated-protection against CPT-induced apoptosis, indicating that the ERK1/2 pathway plays a role in the antiapoptotic effects of PKA activation. $\boldsymbol{E}$, Neurons were transfected as in Figure 3. Three days after 0.001 . .001. ns, Nonsignificant. $\boldsymbol{F}$, Our data support a model in which the antiapoptotic activity of KSR1-ERK1/2 is as a convergence poin for protective signaling by BDNF/TrkB and CAMP/PKA.

indicate that the ERK1/2 pathway protecting against TD involves a scaffolding protein(s) different from KSR1.

At present, it is unclear what are the ERK1/2 substrates that are critical for KSR1-ERK1/2-mediated suppression of DNA damage-induced apoptosis. The p90 ribosomal S6 kinase (RSK) has been implicated as a mediator of antiapoptotic ERK1/2 activity in BDNF-treated cerebellar granule neurons that were challenged with TD (Bonni et al., 1999). However, in hippocampal slices, KSR1 appeared to direct ERK1/2 activity toward the Kv4.2 potassium channel subunit but not p90 RSK (Shalin et al., 2006).If similar substrate preferences occur for KSR1-regulated ERK1/2 in cortical neurons, this would argue against the role of p90 RSK in neuroprotection against DNA damage. Indeed, our preliminary experiments indicated that BDNF inhibition of CPT-induced apoptosis was not disturbed by blocking either $\mathrm{p} 90$ RSK1 or RSK2 or RSK3 (Kharebava and Hetman, unpublished observations). However, inhibition of RSK1 and RSK2 removed BDNF protection against TD (Kharebava, Kalita, and Hetman, 
unpublished observations). The role of ERK1/2-mediated phosphorylation of Kv4.2 in antiapoptotic signaling by ERK1/2 remains to be tested.

Our results indicate that PKA activates neuronal signaling by ERK1/2 that is regulated by KSR1. The activation involved MKK1/2 but not TrkB BDNF receptor. This suggests that in cultured cortical neurons, the coupling between PKA and ERK is by a different mechanism(s) than in hippocampal or retinal ganglion cells in which PKA-mediated enhancement of TrkB signaling has been implicated as a link between cAMP and ERK1/2 (Meyer-Franke et al., 1998; Ji et al., 2005). Also, PKA-mediated inactivation of the ERK1/2 striatal-enriched protein tyrosine phosphatase (STEP) is not a likely mechanism underlying cAMP to ERK1/2 signaling in cortical neurons (Paul et al., 2003). Arguments against that possibility include robust activation of MKK1/2 and unaltered rate of ERK1/2 dephosphorylation in forskolin-treated neurons (Fig. 3 and data not shown). If inhibition of ERK1/2 phosphatase/phosphatases will be a major mechanism underlying cAMP-mediated activation of ERK1/2, one could expect little effect on the ERK1/2 kinase and MKK1/2 and slower dephosphorylation of pERK1/2. The contribution of previously reported mechanisms of PKA/ERK1/2 cross talk including PKA-mediated activation of a small GTPase, Rap1, and a MKK1/2 kinase, B-Raf, remain to be tested (Vossler et al., 1997). Of note, KSR1 knockdown only partially removed forskolin protection against CPT that was completely blocked if ERK1/2 or PKA were inhibited. Therefore, it is possible that in addition to KSR1, other scaffolding proteins contribute to antigenotoxic signaling by PKA-ERK1/2.

Our results indicate that stimulation of cAMP-PKA-ERK1/2 signaling offers antiapoptoptotic neuroprotection against DNA damage by the anticancer drug CPT. We observed similar protective effects in neurons treated with the anticancer drug cisplatin, the high neurotoxicity of which is at least in part attributable to DNA damage (Szatmari, J. Zheng, and Hetman, unpublished observations). Therefore, agents that activate neuronal cAMP signaling may be considered as potential remedies against neurotoxicity of anticancer therapeutics that damage DNA. Importantly, cAMP signaling may be a better target than BDNF/TrkB to therapeutically modify antigenotoxic signaling by ERK1/2. First, activation of a neuroprotective cAMP/PKA pathway could be obtained using several small molecule drugs including inhibitors of cAMP-specific phosphodiesterases or agonists of G-proteincoupled receptors (McCullough et al., 2004; Pearse et al., 2004). Second, the presence of neurotrophin receptors including TrkB has been reported on tumor cells and implicated in malignancy (Desmet and Peeper, 2006). However, cAMP may have anticancer effects, at least in some neoplastic diseases (Chen et al., 1998; Lerner and Epstein, 2006). Therefore, cAMP signaling may be more selective than BDNF/TrkB in protecting normal components of the nervous system without compromising cancer cell killing.

In summary, we identified KSR1 as a part of the neuronal signaling network activated by BDNF or cAMP. KSR1 contributed to BDNF or CAMP activation of ERK1/2 that was required for suppression of DNA damage-induced neuronal apoptosis. Thus, KSR1 is a novel transducer on the antiapoptotic ERK1/2 pathway that supports neuronal survival. In addition, our results indicate that activation of cAMP signaling may offer therapeutic opportunities against neurotoxicity of DNA-damaging anticancer agents.

\section{References}

Adams JP, Sweatt JD (2002) Molecular psychology: roles for the ERK MAP kinase cascade in memory. Annu Rev Pharmacol Toxicol 42:135-163.

Anderson CN, Tolkovsky AM (1999) A role for MAPK/ERK in sympathetic neuron survival: protection against a p53-dependent, JNK-independent induction of apoptosis by cytosine arabinoside. J Neurosci 19:664-673.

Bonni A, Brunet A, West AE, Datta SR, Takasu MA, Greenberg ME (1999) Cell survival promoted by the Ras-MAPK signaling pathway by transcription-dependent and -independent mechanisms [see comments]. Science 286:1358-1362.

Brummelkamp TR, Bernards R, Agami R (2002) A system for stable expression of short interfering RNAs in mammalian cells. Science 296:550-553.

Cacace AM, Michaud NR, Therrien M, Mathes K, Copeland T, Rubin GM, Morrison DK (1999) Identification of constitutive and ras-inducible phosphorylation sites of KSR: implications for 14-3-3 binding, mitogenactivated protein kinase binding, and KSR overexpression. Mol Cell Biol 19:229-240.

Chen TC, Hinton DR, Zidovetzki R, Hofman FM (1998) Upregulation of the cAMP/PKA pathway inhibits proliferation, induces differentiation, and leads to apoptosis in malignant gliomas. Lab Invest 78:165-174.

Cherrington JM, Mocarski ES (1989) Human cytomegalovirus iel transactivates the $\alpha$ promoter-enhancer via an 18-base-pair repeat element. J Virol 63:1435-1440.

del Peso L, Gonzalez-Garcia M, Page C, Herrera R, Nunez G (1997) Interleukin-3-induced phosphorylation of BAD through the protein kinase Akt. Science 278:687-689.

Desmet CJ, Peeper DS (2006) The neurotrophic receptor TrkB: a drug target in anti-cancer therapy? Cell Mol Life Sci 63:755-759.

Enserink JM, Christensen AE, de Rooij J, van Triest M, Schwede F, Genieser HG, Doskeland SO, Blank JL, Bos JL (2002) A novel Epac-specific cAMP analogue demonstrates independent regulation of Rapl and ERK. Nat Cell Biol 4:901-906.

Geller HM, Cheng KY, Goldsmith NK, Romero AA, Zhang AL, Morris EJ, Grandison L (2001) Oxidative stress mediates neuronal DNA damage and apoptosis in response to cytosine arabinoside. J Neurochem 78:265-275.

Giblett SM, Lloyd DJ, Light Y, Marais R, Pritchard CA (2002) Expression of kinase suppressor of Ras in the normal adult and embryonic mouse. Cell Growth Differ 13:307-313.

Gonzalez-Zulueta M, Feldman AB, Klesse LJ, Kalb RG, Dillman JF, Parada LF, Dawson TM, Dawson VL (2000) Requirement for nitric oxide activation of p21(ras)/extracellular regulated kinase in neuronal ischemic preconditioning. Proc Natl Acad Sci USA 97:436-441.

Gozdz A, Habas A, Jaworski J, Zielinska M, Albrecht J, Chlystun M, Jalili A, Hetman M (2003) Role of N-Methyl-D-aspartate receptors in the neuroprotective activation of extracellular signal-regulated kinase $1 / 2$ by cisplatin. J Biol Chem 278:43663-43671.

Groom LA, Sneddon AA, Alessi DR, Dowd S, Keyse SM (1996) Differential regulation of the MAP, SAP and RK/p38 kinases by Pyst1, a novel cytosolic dual-specificity phosphatase. EMBO J 15:3621-3632.

Habas A, Kharebava G, Szatmari E, Hetman M (2006) NMDA neuroprotection against a phosphatidylinositol-3 kinase inhibitor, LY294002 by NR2B-mediated suppression of glycogen synthase kinase-3beta-induced apoptosis. J Neurochem 96:335-348.

Hetman M, Gozdz A (2004) Role of extracellular signal regulated kinases 1 and 2 in neuronal survival. Eur J Biochem 271:2050-2055.

Hetman M, Kanning K, Smith-Cavanaugh JE, Xia Z (1999) Neuroprotection by brain-derived neurotrophic factor is mediated by extracellularsignal-regulated kinase and phosphatidylinositol-3 kinase. J Biol Chem 274:22569-22580.

Hetman M, Hsuan SL, Habas A, Higgins MJ, Xia Z (2002) ERK1/2 antagonizes glycogen synthase kinase-3beta -induced apoptosis in cortical neurons. J Biol Chem 277:49577-49584.

Jacobson MD, Weil M, Raff MC (1997) Programmed cell death in animal development. Cell 88:347-354.

Ji Y, Pang PT, Feng L, Lu B (2005) Cyclic AMP controls BDNF-induced TrkB phosphorylation and dendritic spine formation in mature hippocampal neurons. Nat Neurosci 8:164-172.

Kaplan DR, Miller FD (2000) Neurotrophin signal transduction in the nervous system. Curr Opin Neurobiol 10:381-391.

Koh DW, Dawson TM, Dawson VL (2005) Mediation of cell death by poly(ADP-ribose) polymerase-1. Pharmacol Res 52:5-14. 
Kruman II, Kumaravel TS, Lohani A, Pedersen WA, Cutler RG, Kruman Y, Haughey N, Lee J, Evans M, Mattson MP (2002) Folic acid deficiency and homocysteine impair DNA repair in hippocampal neurons and sensitize them to amyloid toxicity in experimental models of Alzheimer's disease. J Neurosci 22:1752-1762.

Lee B, Butcher GQ, Hoyt KR, Impey S, Obrietan K (2005) Activitydependent neuroprotection and cAMP response element-binding protein (CREB): kinase coupling, stimulus intensity, and temporal regulation of CREB phosphorylation at serine 133. J Neurosci 25:1137-1148.

Lerner A, Epstein PM (2006) Cyclic nucleotide phosphodiesterases as targets for treatment of haematological malignancies. Biochem J 393:21-41.

Markus A, Patel TD, Snider WD (2002) Neurotrophic factors and axonal growth. Curr Opin Neurobiol 12:523-531.

McCullough L, Wu L, Haughey N, Liang X, Hand T, Wang Q, Breyer RM, Andreasson K (2004) Neuroprotective function of the PGE2 EP2 receptor in cerebral ischemia. J Neurosci 24:257-268.

McDonald ES, Randon KR, Knight A, Windebank AJ (2005) Cisplatin preferentially binds to DNA in dorsal root ganglion neurons in vitro and in vivo: a potential mechanism for neurotoxicity. Neurobiol Dis 18:305-313.

McKay MM, Morrison DK (2007) Caspase-dependent cleavage disrupts the ERK cascade scaffolding function of KSR1. J Biol Chem 282:26225-26234.

Meyer-Franke A, Wilkinson GA, Kruttgen A, Hu M, Munro E, Hanson MG, Reichardt LF, Barres BA (1998) Depolarization and cAMP elevation rapidly recruit TrkB to the plasma membrane of CNS neurons. Neuron 21:681-693.

Morrison DK, Davis RJ (2003) Regulation of MAP kinase signaling modules by scaffold proteins in mammals. Annu Rev Cell Dev Biol 19:91-118.

Muller J, Cacace AM, Lyons WE, McGill CB, Morrison DK (2000) Identification of B-KSR1, a novel brain-specific isoform of KSR1 that functions in neuronal signaling. Mol Cell Biol 20:5529-5539.

Muller J, Ory S, Copeland T, Piwnica-Worms H, Morrison DK (2001) C-TAK1 regulates Ras signaling by phosphorylating the MAPK scaffold, KSR1. Mol Cell 8:983-993.

Ory S, Zhou M, Conrads TP, Veenstra TD, Morrison DK (2003) Protein phosphatase $2 \mathrm{~A}$ positively regulates Ras signaling by dephosphorylating KSR1 and Raf-1 on critical 14-3-3 binding sites. Curr Biol 13:1356-1364.

Paul S, Nairn AC, Wang P, Lombroso PJ (2003) NMDA-mediated activation of the tyrosine phosphatase STEP regulates the duration of ERK signaling. Nat Neurosci 6:34-42.

Pearse DD, Pereira FC, Marcillo AE, Bates ML, Berrocal YA, Filbin MT,
Bunge MB (2004) cAMP and Schwann cells promote axonal growth and functional recovery after spinal cord injury. Nat Med 10:610-616.

Pearson G, Robinson F, Beers Gibson T, Xu BE, Karandikar M, Berman K, Cobb MH (2001) Mitogen-activated protein (MAP) kinase pathways: regulation and physiological functions. Endocr Rev 22:153-183.

Rolig RL, McKinnon PJ (2000) Linking DNA damage and neurodegeneration. Trends Neurosci 23:417-424.

Shalin SC, Hernandez CM, Dougherty MK, Morrison DK, Sweatt JD (2006) Kinase suppressor of Ras1 compartmentalizes hippocampal signal transduction and subserves synaptic plasticity and memory formation. Neuron 50:765-779.

Torii S, Kusakabe M, Yamamoto T, Maekawa M, Nishida E (2004) Sef is a spatial regulator for Ras/MAP kinase signaling. Dev Cell 7:33-44.

Vantaggiato C, Formentini I, Bondanza A, Bonini C, Naldini L, Brambilla R (2006) ERK1 and ERK2 mitogen-activated protein kinases affect Rasdependent cell signaling differentially. J Biol 5:14.

Villalba M, Journot L (1997) Pituitary adenylate cyclase-activating polypeptide (PACAP-38) protects cerebellar granule neurons from apoptosis by activating the mitogen-activated protein kinase (MAP kinase) pathway. J Neurosci 17:83-90.

Vossler MR, Yao H, York RD, Pan MG, Rim CS, Stork PJ (1997) cAMP activates MAP kinase and Elk-1 through a B-Raf- and Rap1-dependent pathway. Cell 89:73-82.

Wang J, Xiong S, Xie C, Markesbery WR, Lovell MA (2005) Increased oxidative damage in nuclear and mitochondrial DNA in Alzheimer's disease. J Neurochem 93:953-962.

Wiley JC, Wailes LA, Idzerda RL, McKnight GS (1999) Role of regulatory subunits and protein kinase inhibitor (PKI) in determining nuclear localization and activity of the catalytic subunit of protein kinase A. J Biol Chem 274:6381-6387.

Yan F, John SK, Polk DB (2001) Kinase suppressor of Ras determines survival of intestinal epithelial cells exposed to tumor necrosis factor. Cancer Res 61:8668-8675.

Yan F, John SK, Wilson G, Jones DS, Washington MK, Polk DB (2004) Kinase suppressor of Ras-1 protects intestinal epithelium from cytokinemediated apoptosis during inflammation. J Clin Invest 114:1272-1280.

Yun MS, Kim SE, Jeon SH, Lee JS, Choi KY (2005) Both ERK and Wnt/betacatenin pathways are involved in Wnt3a-induced proliferation. J Cell Sci 118:313-322.

Zhu D, Wu X, Strauss KI, Lipsky RH, Qureshi Z, Terhakopian A, Novelli A, Banaudha K, Marini AM (2005) N-methyl-D-aspartate and TrkB receptors protect neurons against glutamate excitotoxicity through an extracellular signal-regulated kinase pathway. J Neurosci Res 80:104-113. 